\section{The effects of perspective alterations on apparent size and distance scales}

\author{
JULIET M. VOGEL \\ Harvard University, Cambridge, Massachusetts 02138 \\ and \\ MARTHA TEGHTSOONIAN* \\ Smith College, Northampton, Massachusetts 01060
}

Ss made magnitude estimations first of the areas, then of the distances, of four circles presented at five distances within an enclosed box $9 \mathrm{ft}$ long. There were three viewing situations: the box had parallel walls, converging walls, or diverging walls. The variations in perspective cues influenced the apparent distance scales: with converging and parallel walls, apparent distance grew as the 1.4 power of physical distance; with diverging walls, it grew as the .95 power. Apparent area was related to distance differently in the three conditions: with converging walls, apparent area increased with distance; with parallel and diverging walls, apparent area decreased with distance. Apparent area was thus not related to changes in physical distance as the apparent distance effects would predict. In each condition, the ratio of apparent area to apparent distance $\left(S^{\prime} / D^{\prime}\right)$ was a monotonic increasing function of retinal angle $\theta$, but the rate of growth varied among the three conditions. The failure of $\theta D^{\prime}$ to define a unique value of $S^{\prime}$ across conditions indicates that the size-distance invariance hypothesis is inadequate to predict the outcome of this experiment.

The tendency of Os to see an object as a constant size when changes in the object's distance produce changes in retinal image size is called size constancy. Size-distance invariance hypotheses, one class of explanations invoked to account for size constancy, have the common feature that the observer is said to use distance judgments to "correct" his impressions of size. In investigating the influence of apparent distance on apparent size, one way for $E$ to manipulate apparent distance is to change physical distance. If he presents objects at different distances, however, he must also vary their physical sizes to maintain a constant retinal size; if he holds physical size constant, he will produce retinal size changes. To test size-distance invariance hypotheses, a method which allows the manipulation of the apparent distance of an object while holding both its physical and retinal sizes constant is desirable. The study to be reported here achieved this end by providing three sets of perspective cues to distance: three boxes were constructed whose walls were parallel to each other, diverged from each other (relative to S's eye), or converged. If the manipulated perspective cues influenced apparent

\footnotetext{
*The research reported here was undertaken by the first author as part of the honors program, Department of Psychology, Smith College; the second author was her advisor. The manuscript was prepared while the second author was a visitor in the Laboratory of Psychophysics, Harvard University. We thank J. Hay for discussion and criticism of the experiment and $R$. Teghtsoonian for comments on the manuseript.
}

distance, then an object placed at a given distance from $\mathbf{S}$ would have the same retinal size but would appear to be at different distances in the three boxes. That the perspective changes introduced by diverging and converging walls are effective in altering apparent distance was shown by Blessing, Landauer, and Coltheart (1967) in a study of size-distance invariance.

A second feature of the study to be reported is that it varied both size and distance parametrically: within each perspective condition, several distances were presented as targets and several areas were presented at each distance. Ss made magnitude estimations of the areas and distances. Teghtsoonian and Teghtsoonian (1970) have shown that in a natural viewing situation, this magnitude estimation procedure yields orderly size and distance scales. Judgments were analyzed to see if (1) the perspective alterations produced different apparent distance scales, (2) apparent area scales were affected by the perspective alterations, and (3) apparent distance and apparent area scales were related as a size-distance invariance hypothesis would predict.

\section{METHOD}

Subjects

The Ss were 36 female college undergraduates who had had no previous experience with this type of experiment. All Ss normally using glasses for distance viewing wore their glasses during the experiment. Twelve Ss participated in each perspective condition.
Apparatus

Two boxes were used, one to provide the two altered perspectives and a second, rectangular, box which served as a control.

Conditions were labeled according to the slant of the side walls from the $S$ to the back of the box used-the side walls for the "converging" (C) condition would meet if extended behind the rear wall, side walls for the "parallel" (P) condition would remain parallel, and side walls for the "diverging" (D) condition would become increasingly far apart.

The boxes were $9 \mathrm{ft}$ long. The top and viewing ends of each box were open. The interiors were painted dull gray. The box for Condition $P$ was $3 \mathrm{ft}$ wide and $2 \mathrm{ft}$ high. For Condition $D$, Ss used the second box, looking through its open small end, which was $1 \mathrm{ft}$ on each side, toward the closed larger end, which was $3 \mathrm{ft}$ on each side. The floor sloped downward from the viewing end to the back of the box. For Condition $C$, the small end was closed by inserting a movable panel and the langer end was opened for viewing. The floor sloped upward from the viewing end. The box used for the $\mathrm{C}$ and $\mathrm{D}$ conditions is depicted in Fig. 1.

For each condition, the appropriate box was set up in a room illuminated to minimize shadows in the box. A screen against the viewing end prevented S's seeing the exterior of the box. A viewer was attached to a headrest and extended through the screen at the center of the viewing end of the box. It fit over both eyes and defined a visual field the shape of a flattened ellipse. The headrest and viewer kept S's head 6 in. from the box and, for Conditions $D$ and $P$, prevented S's seeing the top of the box.

Stimuli were four circles cut from black photo mount. Their areas were $17.5,29.6,48.7$, and 82.3 in. ${ }^{2}$ The circles were presented on the floor of the box standing parallel to the rear wall at distances of $3,4.5,6,7.5$, and $9 \mathrm{ft}$ from $S$.

The standard for distance judgments was a 1-ft horizontal black line directly above the viewer on the screen in front of the box. No standard was used for size judgments.

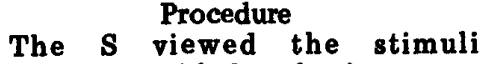
monocularly with her dominant eye, determined by sighting tests at the beginning of the experimental session; her other eye was covered with a patch.

All Ss judged size before distance. The $S$ viewed an empty box while $E$ read the size-judgment instructions. Thereafter, $s$ viewed the box only 


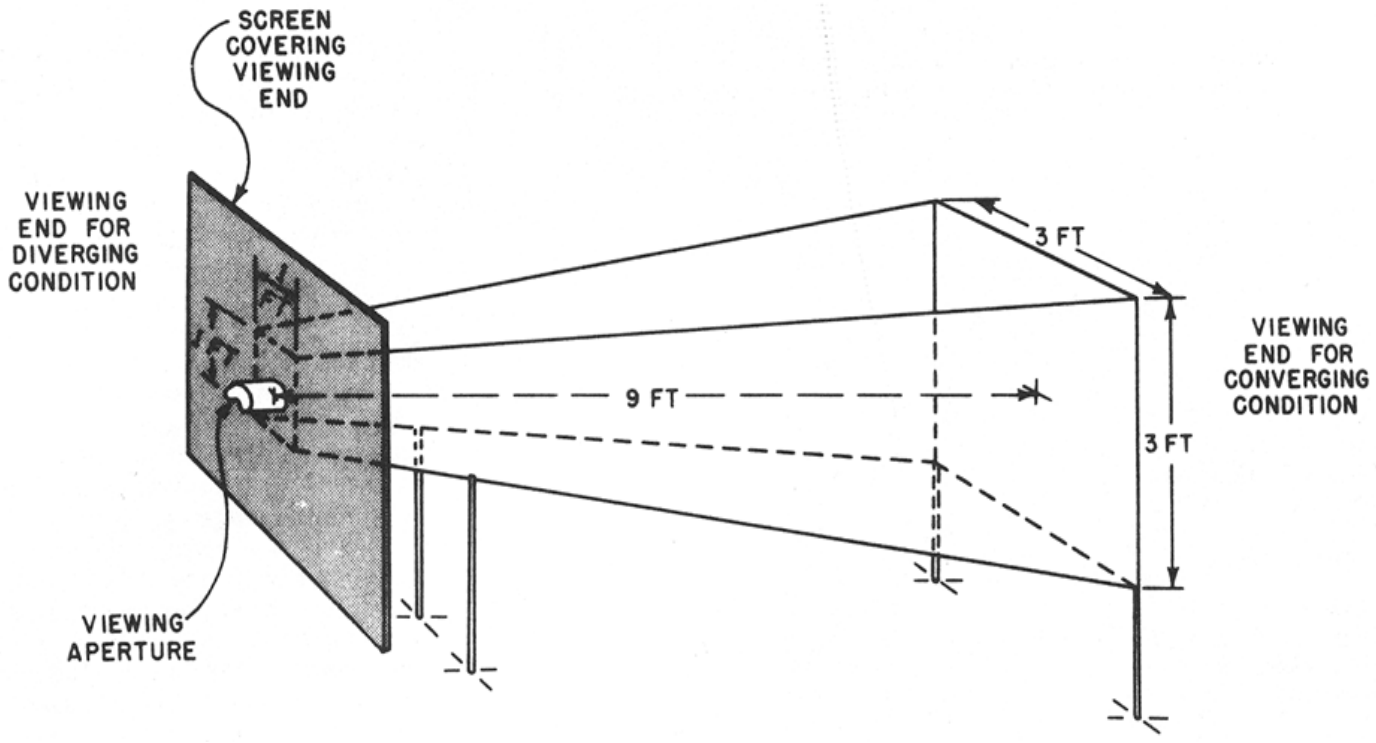

Fig. 1. Perspective box used for $C$ and $D$. $S$ viewed from end on left for Condition $D$ and from end on right for Condition $\mathrm{C}$; the end panels were removable.

when a stimulus was in position, closing her eyes while stimuli were being changed.

The size-judgment instructions were as follows: "Try not to move your head during the experiment. I'm going to show you some circles of different areas. They will be displayed at different distances from you in this box. I want you to tell me how large they are in terms of area. Assign to the first circle the number which seems to you to represent best its area. You may use any number you wish: for example, a number such as 85 or 217 or a number less than one such as $2 / 3$ or $1 / 10$. Then to each successive circle assign the number which seems to you to best represent its area in comparison to the first circle."

Each circle was presented once at each of five distances. A different, randomly generated order was used for each $S$.

Immediately after $S$ completed the size judgments, $E$ read the following distance-judgment instructions: "I'm going to show you some more circles, and I'd like you to estimate how far away from you they are. Make your judgments in feet. To give you an idea how long a foot is, there is a black line $1 \mathrm{ft}$ long on the screen at the front of the box. Take a good look at the line now, but do not look back at it once you begin to make your judgments."

The largest and smallest circles were presented at each of the five distances used for the size judgments. A different, randomly generated order was used for each $\mathbf{S}$

After completing the judgments, $S$ went into another room and filled out a questionnaire about the experiment, including a checklist about the shape of the box.
RESULTS AND DISCUSSION

The Effect of Perspective Alteration on Apparent Distance

For each S, least-squares lines were fitted to log distance judgments as a function of $\log$ distance. The resulting functions yield, for each $S$, two "scores"-a slope constant and an additive constant-which may be interpreted as the exponent and scale factor of the individual power functions relating apparent to physical distance. Figure 2 shows the mean distance functions, which are: converging, $\psi_{d}=.32 d^{1.42}$; parallel, $\psi_{\mathrm{d}}=.41 \mathrm{~d}^{1.40}$; and diverging, $\psi_{\mathrm{d}}=$ $.81 \mathrm{~d}^{0.95}$, where $\mathrm{d}$ is distance in feet. The mean coefficients of determination for individual power functions are $.96, .98$, and .97 for $C$, $P$, and $D$, respectively; the power functions are good fits to the individual judgments.

Thus, the perspective alterations influenced the rate at which apparent distance grows. An analysis of variance of individual distance exponents indicated that they differ significantly across perspective conditions $(F=4.55$, df $=2,33, \quad p<.05)$. The exponents for $P$ and $C$, which do not differ significantly from each other, differ significantly from the exponent for $D$. In $P$ and $C$, ratios of distances are overestimated: the exponent of 1.4 means that doubling the distance increases apparent distance by a factor of about 2.6. In $D$, ratios of distances are underestimated: the exponent of .95 means that doubling the distance increases apparent distance by a factor of about 1.9 .

$P$, designed as a control condition, produced a distance scale comparable to those for normal indoor viewing. The exponents near 1.4 for $P$ and $C$ are consistent with the findings of Künnapas (1960) and Teghtsoonian and Teghtsoonian (1969) that apparent distance indoors is an accelerating function of physical

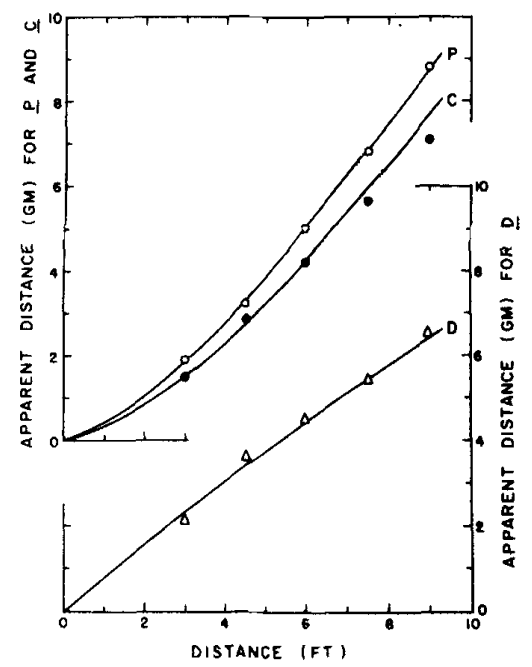

Fig. 2. Apparent distance as a function of physical distance in three perspective conditions. Shown are geometric means of magnitude estimations of the apparent distance of targets placed in perspective boxes with converging (C), parallel $(P)$, or diverging (D) walls; $N=12$ for each condition. The curves represent the best-fitting power functions, with exponent 1.40 for $P, 1.42$ for $C$, and .95 for $D$. The ordinate for $P$ and $C$ is at the left; the ordinate for $D$ is at the right and is displaced relative to that for $P$ and $C$ in order to display the points clearly. 
Table 1

Analysis of Variance of Log Magnitude Estimations of Circle Area for Four Areas at Five Distances Within Three Perspective Conditions

\begin{tabular}{|c|c|c|c|c|}
\hline Source & df & SS & MS & $\mathbf{F}$ \\
\hline Between Ss & & 214.900 & & \\
\hline A (perspective) & 2 & 15.074 & 7.537 & 1.245 \\
\hline S8/A & 33 & 199.825 & 6.055 & \\
\hline Within Ss & & 46.300 & & \\
\hline B (area) & 3 & 27.454 & 9.151 & $272.360 \dagger$ \\
\hline $\operatorname{Lin}(\log B)$ & (1) & 27.444 & 27.444 & $349.160 t$ \\
\hline Dev Lin(log B) & (2) & 0.010 & .005 & $<1.0$ \\
\hline $\mathbf{A B}$ & 6 & 0.098 & .016 & $<\mathbf{1 . 0}$ \\
\hline B by Ss/A & 99 & $\mathbf{3 . 2 3 8}$ & .034 & \\
\hline Lin $(\log B)$ by $S s / A$ & (33) & 2.594 & .079 & \\
\hline Dev Lin(log B) by Ss/A & (66) & 0.643 & .010 & \\
\hline C (distance) & 4 & 1.405 & .351 & $9.340 t$ \\
\hline $\operatorname{Lin}(C)$ & (1) & 1.350 & 1.350 & $15.757 t$ \\
\hline Dev Lin(C) & (3) & 0.054 & .018 & $<1.0$ \\
\hline $\mathrm{AC}$ & 8 & $\mathbf{3 . 4 1 2}$ & .426 & $11.343 \dagger$ \\
\hline $\operatorname{Lin}(C)$ by $A$ & (2) & $\mathbf{3 . 3 7 0}$ & 1.685 & $19.693 \dagger$ \\
\hline $\operatorname{Lin}(C) / p$ and $c$ vs $\operatorname{Lin}(C) / d^{*}$ & [1] & 2.572 & 2.572 & $29.434 t$ \\
\hline $\operatorname{Lin}(C) / p$ vs $\operatorname{Lin}(C) / c^{*}$ & [1] & 0.797 & .797 & $9.121 * *$ \\
\hline Dev Lin(C) by A & (6) & 0.042 & .007 & $<1.0$ \\
\hline C by $\mathrm{Ss} / \mathrm{A}$ & 132 & 4.961 & .038 & \\
\hline $\operatorname{Lin}(\mathrm{C})$ by Ss/A & (33) & 2.823 & .086 & \\
\hline Dev Lin(C) by Ss/A & (99) & 2.148 & .022 & \\
\hline BC & 12 & 0.226 & .019 & 1.488 \\
\hline ABC & 24 & 0.384 & .016 & 1.260 \\
\hline BC by $\mathrm{Ss} / \mathrm{A}$ & 396 & 5.032 & .013 & \\
\hline
\end{tabular}

$* p$, $c$, and $d$ refer to the parallel, converging, and diverging perspective conditions $* * p<.01$, tp $<.001$

distance, and these exponents are very close to the value of 1.47 obtained for the most comparable range of distances in those studies, 3 to $19 \mathrm{ft}$ (Künnapas, 1960). The exponent of .95 for $\mathrm{D}$ was a marked deviation from this value.

An analysis of variance of individual scale factors indicated that they do not differ significantly across perspective conditions $(F=1.64$, df $=2,33, p>.05$ ).

\section{The Effect of Perspective Alteration on Apparent Area}

Area judgments were transformed to their logarithms, and an analysis of variance, summarized in Table 1, was performed on these data. (The logarithmic transformation allowed us to compare Ss with respect to the ratios between each S's judgments regardless of the number she assigned to the first stimulus.) The data were examined, first, to ascertain the nature of the apparent-area scales, and, second, to discover the effect of distance.

Apparent area scales. On the basis of previous work, we expected that apparent area would be a power function of circle area and that the exponent of the power function would be unaffected by distance or by perspective alteration. These expectations were confirmed. A trend analysis (summarized in Table 1) indicated that the significant effect of circle area on area judgments is accounted for by the linear relation (slope $=.81$ ) between log area judgment and log circle area. This confirms the power relation reported by Stevens and Guirao (1963) and Teghtsoonian (1965), and the exponent is close to that of .76 reported by Teghtsoonian (1965) when Ss were asked to make magnitude estimations of apparent area of circles. ${ }^{1}$ The exponent of the power function is the same at all distances and in all perspective conditions (as shown by the absence of either a significant Distance by Area interaction or a significant Perspective by Area interaction). This relative robustness of the apparent-area exponent was noted by Teghtsoonian and Teghtsoonian (1970), who found the exponent invariant at distances from 5 to $45 \mathrm{ft}$.

Perspective condition and the relation between apparent area and physical distance. To see whether the perspective alterations influenced apparent area, the data were replotted to show geometric means of the area judgments as a function of distance for the three perspective conditions, as shown in Fig. 3. The analysis of variance (Table 1) showed that distance has a significant effect on area judgments and that the interaction between distance and perspective is significant. Trend analyses (Table 1) indicated that the significant distance effect can be accounted for by the linear relation between log area judgments and distance and that the perspective-distance interaction can be accounted for by the significant differences among the linear slopes of these functions. Contrasts of the trends for different conditions indicated that $D$ differs significantly from $P$ and $C$ and that $P$ and $C$ differ significantly from each other in linear slope. The linear relationship between $\log$ apparent area and distance suggested by this analysis is equivalent to apparent area's being an exponential function of distance. The smooth curves in Fig. 3 represent these functions, with exponents as indicated by the trend analyses.

It is clear that apparent area changed as a function of distance in each perspective condition but that the perspective condition determined the nature of the change. When walls were converging, apparent area was an increasing and slightly positively accelerating function of physical distance: over a difference in physical distance of $6 \mathrm{ft}$, this amounted to a $16 \%$ increase in apparent area for an object of given physical size. When walls were parallel, apparent area was a decreasing and slightly negatively accelerating function of distance: a 6-ft difference in distance corresponded to a $21 \%$ decrease in apparent area. When walls were diverging, there was a more sharply decreasing, although negatively

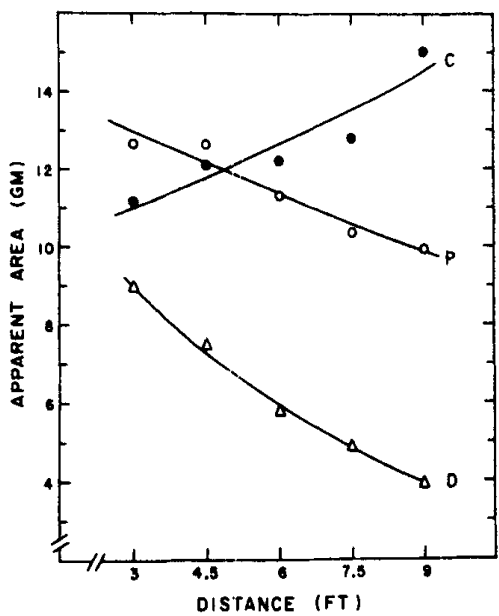

Fig. 3. Apparent area as a function of physical distance in three perspective conditions. Shown are geometric means of magnitude estimations of the apparent area of circles placed at different distances in perspective boxes with converging $(C)$, parallel $(P)$, and diverging (D) walls; $\mathrm{N}=12$ for each condition. The lines represent the best-fitting exponential functions: apparent area is proportional to $10^{+.02 d}$, where $d$ is distance in feet, for $C ; 10^{-}-02 d$ for $P$; and 10-.06d for $D$. 


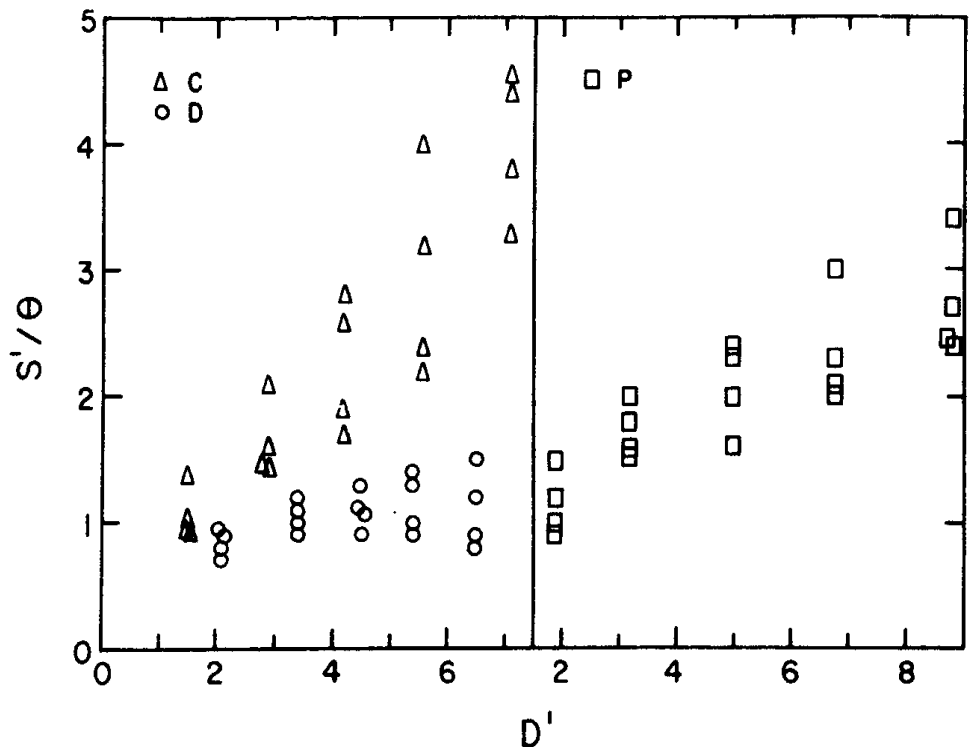

Fig. 4. Ratio of apparent size of a target to retinal angle of the target as a function of apparent distance at which target appeared, for Conditions $D, C$, and $P$. $S^{\prime}$ represents geometric means of magnitude estimations of area, $D^{\prime}$ represents geometric means of magnitude estimations of distance ( $N=12$ in each condition). $\theta$ is approximated by considering that $\tan \theta / 2=$ (target diameter)/2(target distance).

accelerating, function: a 6-ft difference in distance corresponded to a $56 \%$ decrease in apparent area.

Perspective condition and the relation between apparent area and apparent distance. (1) Grouped data. While each perspective condition was characterized by a distinctive relation between apparent area and distance, these relations were not predicted by the characteristic apparent distance functions. Thus, the distance exponent for $P$ and $C$ is the same; but in one case, $C$, an object of fixed area appears to get larger when it is moved further away, while in the other case, $P$, an object of fixed area appears to get smaller.

The relation between apparent size and apparent distance is shown directly in Fig. 4, in which the ratio of apparent size to retinal size is plotted as a function of apparent distance for the three conditions. In each condition, $S^{\prime} / \theta$ is a monotonic increasing function of $D^{\prime} .^{2}$ However, if the product $\theta \mathrm{D}^{\prime}$ determined a unique $S^{\prime}$, as demanded by an invariance hypothesis, all the points in Fig. 4 would fall along a single function, whereas three functions, one for each viewing condition, are defined. As Gogel, Wist, and Harker (1963) pointed out, "If $\mathrm{K}$ varies as a function of situations... [the size-distance invariance hypothesis $\left.S^{\prime}=K \theta D^{\prime}\right]$ loses much of its generality." If, in the present study, $S$ used distance judgments to arrive at his impressions of size, his way of doing this depended upon the background against which the stimuli were presented.

These data add to the evidence cited by Epstein, Park, and Casey (1961) that apparent-distance changes are not consistently accompanied by the apparent-size changes required by a SDIH, and to the data presented by Gogel, Wist, and Harker (1963) showing that $\mathrm{K}$ may vary as a function of viewing condition.

The results from this study may be compared to those from other studies in which $D^{\prime}$ was controlled by manipulating pictorial cues to depth. McDonald and O'Hara (1964) changed $D^{\prime}$ by manipulating optical contact for a single value of $\theta$; they found that apparent size changed in the direction predicted by a SDIH but that the value of $\mathbf{S}^{\prime}$ was smaller than that predicted by the product $\theta \mathrm{D}^{\prime}$. Blessing, Coltheart, and Landauer (1967) cues (in a manner similar to that of the present study) and, plotting results for individual $S_{8}$, found that $S^{\prime}=K \theta D^{\prime}$ for a single value of $\theta$. There were differences in the slope $K$ between the two perspective conditions; while not significant, they agreed in direction with the differences in the present study. (It should be noted that Blessing, Coltheart, and Landauer examined what would be two points in the present study, whose parametric nature may make it a more -stringent test of the invariance hypothesis.) The manipulated $D^{\prime}$ by altering perspective evidence, then, while not entirely unambiguous, is consistent with the following statements: (a) for a single viewing condition, changes in apparent distance produced by manipulation of pictorial cues are associated with changes in apparent size such that $\mathbf{S}^{\prime} / \theta$ increases as ' $D$ ' increases; (b) for a single viewing condition, a reasonable approximation of the results is $\mathbf{S}^{\prime} / \theta=\mathrm{KD}^{\prime}$; but (c) a change in viewing conditions may produce a change in $K$, so that, though $\theta$ is invariant across conditions, the relation between apparent size and apparent distance is not.

(2) Individual data. If S's appraisal of apparent distance is the connection between stimulus distance and apparent size, there should be a correlation between the rates at which apparent distance and apparent area grow as functions of physical distance for individual Ss. This relation was studied by finding for each $\mathrm{S}$ the slope of a function relating $\log$ area judgments to distance and correlating this slope with the distance exponent for that $S$. For Ss in the D, C, and $P$ conditions, respectively, the rank-order correlation coefficients were $+.30, .00$, and -.06 , with $p>.05$ in all cases. These results further weaken the argument that distance judgments are used in forming impressions of size, since within each condition the judgments of individual Ss do not conform to the prediction of the size-distance invariance hypothesis, even when $K$ is permitted to vary across conditions.

An Alternative to the SDIH

The present study indicates that if there is an invariant relation between apparent distance and apparent size, the nature of this relation depends on the perceptual context in which a stimulus is viewed, and data for individual Ss fail to support even this weak version of a SDIH. It seems more parsimonious to look for factors in the perceptual context that might influence impressions of size directly and in an invariant way across situations, accepting J. J. Gibson's (1966) argument that perception of objects and their properties is based on cues like "margins, borders, contrasts, ratios, differences, and textures in the array. The stimulating properties of an array thus depend on what is loosely called its structure." In the present experiment, in which cues such as motion parallax and texture of background were minimized, the edges of the box are the most promising source of cues. Factors which might influence perception of size include the relation of the width of the stimulus to the width of the box, the proportion of the back wall covered 
by the stimulus, and the proportion of the stimulus appearing against the back wall vs the proportion appearing against the floor. Future studies might profitably vary such contextual variables systematically to find their effect on apparent size scales.

\section{Adequacy of the Size and Distance}

Judgments to Evaluate a SDIH

If $\mathrm{Ss}$ in normal viewing do "correct" size for distance, as demanded by a SDIH, the present results might be due to $S_{8}$ ' adopting a different attitude in using relations of stimuli to the context provided by the box to judge size. There are no compelling theoretical reasons to believe this must happen; indeed, J. J. Gibson (1966) takes the opposite view, suggesting that the use of relations in the visual array is part of normal perception and that it is the "classical theories of perceptual constancies" (presumably including the use of distance judgment to "perceive" size) which rely on "higher mental processes." The question, then, is not whether our Ss were making cognitive, as opposed to perceptual, responses, but whether they were responding to size and distance as they do in everyday situations. The question is difficult to answer, but two lines of evidence suggest that judgments were similar to those in normal viewing.

The Ss under "objective" size instructions overestimate the size of a far target, whereas under "apparent" size instructions, the size of a far target is estimated accurately (Carlson, 1960) when viewing is binocular. Instructions which produce overestimation of the size of the far target under binocular viewing produce accurate estimation under monocular viewing (Holway \& Boring, 1941). Hence, if "objective" instructions were successful in inducing $S$ deliberately to take distance into account in making size judgments, the result with monocular viewing should be no change in apparent size of target as distance increased. In this study, however, monocular viewing in $P$, the rectangular box, produced a significant decrease in apparent size of target with increasing distance, suggesting that the prevailing set may have corresponded to that which Carieon produced with "apparent" instructions.

Second, if Ss were using factors other than those normally used to judge size and distance, their size and distance scales should have differed from those obtained in normal viewing. With respect to apparent-size/physical-size scales, Ss produced an exponent corresponding to that for Ss estimating apparent size (rather than physical size) under
Table 2

Number of Ss Reporting Each Type of Wall Slant in Three Perapective Conditions

\begin{tabular}{cccc}
\hline & \multicolumn{3}{c}{ Report } \\
\cline { 2 - 4 } $\begin{array}{c}\text { Cond- } \\
\text { tion }\end{array}$ & $\begin{array}{c}\text { Diverg- } \\
\text { ing }\end{array}$ & Parallel & $\begin{array}{c}\text { Converg- } \\
\text { ing }\end{array}$ \\
\hline D & $\mathbf{0}$ & 9 & 3 \\
P & 1 & $8^{*}$ & 3 \\
C & 0 & 2 & $10^{*}$ \\
Total & 1 & 19 & 16 \\
\hline
\end{tabular}

* Correct reports

normal viewing conditions (Teghtsoonian, 1965). With respect to apparent-distance/physical-distance scales, the exponent for $P$ corresponds to that reported by Künnapas (1960) for normal viewing over a similar range of distances, and there is no reason to suspect that $S_{s}$ in $P$ adopted a different attitude from those in $\mathrm{C}$ and D.

\section{Perceived Shape of Box}

For each condition, Ss' reports of the shape of the box are given in Table 2. No $S$ correctly reported the shape of $D$, although most $S$ s in $C$ and $P$ correctly reported box shape. Apparently, the elimination of binocular parallax and the restriction of motion parallax sufficed to conceal from $S$ the shape of the box with diverging walls; however, there were sufficient cues for $S$ to perceive the converging walls. The results for $C$ indicate that the influence of perspective alterations on apparent area scales is not contingent on S's believing that she is viewing a normal rectangular box.

\section{SUMMARY AND CONCLUSIONS}

The perspective alterations produced by converging, parallel, and diverging walls were effective in producing different apparent-distance scales: apparent distance grew as the 1.4 power of physical distance in $C$ and $P$ and as the .95 power in $D$. The perspective alterations were also effective in changing the relation between apparent area and physical distance: log apparent area was related to physical distance in a linear fashion in all three perspective conditions, but the slope of the function was positive for $C$ and negative for $P$ and $D$, markedly so for the latter. The results do not support a size-distance invariance hypothesis. (1) The perspective conditions differ significantly in the relation between distance and apparent distance and differ significantly as well in the relation between distance and apparent area, but it is not possible to predict the latter differences from knowledge of the former. (2) While, within each condition, $S^{\prime} / D^{\prime}$ grows as a monotonic increasing function of $\theta$, the rate of growth differs among the three conditions. (3) For individual Ss within each perspective condition, there is no correlation between the individual exponent of the apparent distance function and the slope of the function relating log apparent area to distance. These outcomes lend indirect support to the alternative hypothesis that distance-related perspective cues influence apparent size directly without operating through an intervening assessment of apparent distance.

\section{REFERENCES}

BLESSING, W. W., LANDAUER, A. A., \& COLTHEART, $M$. The effect of false perspective cues on distance and size-judgments: An examination of the invariance hypothesis. American Joumal of Psychology, 1967, 80, 250-256.

CARLSON, v. R. Overestimation in size-constancy judgments. American Journal of Psychology, 1960, 73, 199-213.

EPSTEIN, W., PARK, J., \& CASEY, A. The current status of the size-distance hy potheses. Pay chological Bulletin, 1961. 58, 491-514.

GIBSON, J. J. Ecological optics. Vision Research, 1961, 1, 253-262.

GOGEL, W. G., WIST, E. R., \& HARKER, G. S. A test of the invariance of the ratio of perceived size to perceived distance. American Journal of Psychology, 1963, 76, 537-553.

HOLWAY, A. H. \& BORING, E. G. Determinants of apparent visual size with distance variant. American Joumal of Pry chology, 1941, 54, 21-37.

KUNNAPAS, T. Scales for subjective distance. Scandinavian Journal of Psychology, 1960, 1, 187-192.

MCDONALD, R. P., \& O'HARA, P. T. Size-distance invariance and perceptual constancy. American Joumal of Psychology, 1964, 77, 276-280.

STEVENS, S. S., \& GUIRAO, M. Subjective scaling of length and area and the matching of length to loudness and brightness. Journal of Experimental Paychology, 1963, 66, 177-186.

TEGHTSOONIAN. M. The judgment of size. American Joumal of Psychology, 1965, 78, 392-402.

T E G H T S O O N I A N, M . \& TEGHTSOONIAN, R. Scaling apparent distance in natural indoor settings. Prychonomic Science, 1969, 16, 281-283.

TEGH TS O O N I A N : R . \& TEGHTSOONIAN, $M$. Effects of size and distance on magnitude estimations of apparent size. American Joumal of Psychology, 1970, 83, 601-612.

\section{NOTES}

1. Teqhteoonian (1965) has shown that when $E$ makes it difficult for $S$ to extimate physical area, the resulting size function is the apparent-area one. We cannot tell what in our procedure or in our $\mathbf{S}$ group made estimated-area instructions ineffective and led our S\& to judge apparent area, but this seems to be what they did.

2. In this study, 's' represents $S^{\prime}$ 's responses to the area of the target figure, whereas in most studies, it represents responses to a linear dimension. Therefore, Fig. 4 is not directly comparable to the usual plots showing the relations among $S^{\prime}$, $\mathbf{D}^{\prime}$, and $\theta$.

(Received for publication October 10 , 1971.) 